\title{
AN INVESTIGATION INTO STUDENT AND QUALIFIED PHYSIOTHERAPISTS DESCRIPTION OF LUNG SOUNDS
}

\begin{abstract}
The ability to accurately describe lung sounds were tested on 146 subjects (quallfied physiotherapists from a tertiary care hospital and third, and fourth year students from three universities). The effect of increased clinical time as well as the nomenclature used was also investigated. The subjects had to recognise six tape recorded lung sounds on a multiple choice answer sheet. It was found that in total the subjects were minimally accurate with a median score of three out of six. The relationship between increasing clinical time and increasing accuracy in determining lung sounds were not significant. Significance was set at $p<0.05$. Comparing third and forth years a p value of 0.0639 was found, while a value 0.3592 was found when comparing forth years and qualified physiotherapists.
\end{abstract}

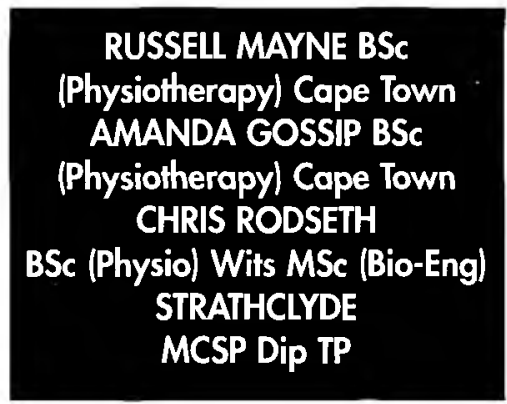
Mean scores did however seem to indicate a trend, as they increased with increasing clinical time. The "Forgacs" nomenclature was used by the majority of subjects tested.

\section{KEY WORDS: PHYSIOTHERAPY, AUSCUCTATION, LUNG SOUNDS, COMMUNITY HEALTH}

\section{INTRODUCTION TO THE PROBLEM}

The recent move of the reconstruction and development program (RDP) towards community based health practices, ${ }^{12}$ has meant the extension of health care to all corners and aspects of the country. The implication of these moves is that access to sophisticated technological equipment is both unfeasible, due to heavy financial restrictions, and impractical due to the location of the clinics. Consequently, community health care givers may need to place greater reliance on auscultation findings when diagnosing chest pathology. It is felt that the ability to accurately identify lung sounds will become vital as a first-line intervention. However, this is not without difficulty. Physicians have always struggled to process the sounds emitted by the lungs in order to gain a better understanding of the changes in structure of the organ. ${ }^{3}$ "They have struggled even more to transmit their understanding of these sounds to naive, willing, but disbelieving students."

Medical and allied medical discipline students are taught to utilise auscultation as a part of chest assessment at all the medical faculties in South Africa. A literature search indicated that no studies on the accuracy of students' auscultation skills have been conducted at any of these medical faculties. The aims of the study were therefore:
1. To investigate the accuracy of physiotherapy students versus qualified physiotherapists in their recognition and description of lung sounds.

2. To investigate whether a relationship exists between the accuracy of lung sound description and the amount of clinical time spent in a respiratory area.

3. To investigate the nomenclature used by student and qualified physiotherapists to describe lung sounds.

\section{METHODOLOGY}

A test tape was produced:

Twenty-one lung sounds were recorded by placing a stethoscope connected to a Sony Pin Microphone (model ECM144) on the patients' chest. This was then connected to an AVICOM tape recorder (Model PA8025).

The recordings were taken from consenting adults at Groote Schuur Hospital, Cape Town. Patients with known lung pathologies were approached. Each patient was auscultated by both researchers and a decision was made as to the quality of the lung sound, which was then recorded. The stethoscope used was binaural and the microphone fitted into the one tube. Simultaneous auscultation of the lung while recording was thus possible, ensuring that an accurate, quality recording was taken.

The folder number, diagnoses and presenting complaints were noted for each patient. The area of auscultation was noted as being over the upper, middle or lower zones as well as anterior or posterior. The sound recordings consisted of at least five breaths. This average duration was suggested by Pasterkamp et al (1987).

The recorded lung sounds were initially analysed subjectively, and although this is perhaps the most inaccurate method of analysis, ${ }^{4}$ it is not inappropri- 


\begin{tabular}{|l|c|c|}
\hline \multicolumn{3}{|l|}{ FIGURE 2 } \\
\hline SAMPLE & 3 & 3.241 .22 \\
\hline Test X & 3 & 2.781 .23 \\
Test Y & 3 & 2.741 .28 \\
3 Year students & 3 & 3.101 .24 \\
4 Year students & 4 & 3.471 .25 \\
Qualified group & 3 & 2.541 .10 \\
3A: University A 3rds & 3 & 2.931 .30 \\
4A: University A 4ths & 3 & 2.470 .84 \\
3B: University B 3rds & 3 & 2.890 .96 \\
4B: University B 4ths & 3 & 3.651 .46 \\
3C: University C 3rds & 4 & 3.501 .29 \\
4C: University C 4ths & 4 & \\
\hline
\end{tabular}

\begin{tabular}{|l|l|}
\hline \multicolumn{2}{|l|}{ FIGURE 3} \\
\hline PERCENTAGE & RATING \\
\hline $0-40$ & Inaccurate \\
$41-50$ & Minimally Accurate \\
$51-60$ & Moderately Accurate \\
$61-75$ & Accurate \\
$76-100$ & Very Accurate \\
\hline
\end{tabular}

ate in this study, as the sounds were more objectively analysed later. Analysis was performed by a senior lecturer at the School of Physiotherapy, University of Cape Town and the control physiotherapist at Groote Schuur hospital. They are both actively involved in the education of students in auscultation. Due to strict criteria that were applied during this subjective verification nine sounds were excluded. Those sounds whose description the specialists did not agree on, and those with unclear recordings were omitted. Seven lung sounds representing a range of auscultation phenomena were chosen by the researchers from the remaining twelve to undergo further screening (see Figure 1).

The spectral and waveform characteristics of these seven sounds were analysed, using a Computerised Speech Lab, Model 4300, Software version 4.x, at the Department of Logopaedics,
University of Cape Town. The sounds used on the test tape were verified by means of objective data from waveform and spectral analysis. Each of the sounds was given a letter name.

A sample sound (example sound) was recorded over the chest of one of the researchers. The peaks in the frequencytime plot fell within the limits set by Graviely et al (1981). These were $604 \mathrm{~Hz}$ $302 \mathrm{~Hz}$ for inspiration and $406 \mathrm{~Hz} 205$ $\mathrm{Hz}$ for expiration, and the conclusion was therefore drawn that the sample sound was a normal breath sound. The time expanded waveform of "Sound A" showed a pattern characteristic of a crackle. ${ }^{7}$ By measuring the initial deflection width (IDW) of the sound it was possible to accurately characterise the sound as either a course or fine crackle. ${ }^{8}$ The IDW for "Sound A" was 1,680 milliseconds 0,580 milliseconds. It was therefore described as a course crackle as it was within the limits set for the IDW by Munataka et al (1981), Burton et al (1984), Mikami et al (1987) and Louden and Murphy (1984). Since there is little objective literature concerning the characteristics of a pleural rub, the validation of "Sound B" was dependent on two factors. The subjective description of the respiratory specialists, and the clinical history of the patient which included the defining characteristics as set by the American Thoracic Society. "Sound C" had a time expanded waveform characteristic of a wheeze.? The wheeze had a frequency of $202 \mathrm{~Hz}$. "Sound D" had a high frequency spike that was heard as a "metallic whistle", 4 this coupled with the subjective analysis determined that "Sound D" was amphoric breathing. "Sound E" was recorded from an asthmatic two hours after being admitted to the Groote Schuur hospital Asthma Room. The time expanded waveform was characteristic of a wheeze' with a frequency of $205 \mathrm{~Hz}$. "Sound F" was a comparison between the right and left anterior upper zones of a patient. The right side had an expanded waveform characteristic of a normal breath sound.' The left side registered considerable interference from the heart sound which is a characteristic of decreased breath sounds. These findings were also consistent with the patient's clinical picture.

\section{TESTING PROTOCOL}

One hundred and forty-six subjects (62 third years, 69 fourth years and 15 qualified physiotherapists) were tested from the sample of 167 subjects. The inclusion criteria were that the subjects had to have had lectures or tuition on lung sounds and in addition, completed a respiratory clinical rotation. Thirty-one third years were excluded as well as three fourth year students who had been involved in the pilot study. The pilot study consisted of the test performed on two fifth year medical students and three fourth year physiotherapy students. The aims of the pilot study were to identify any problems with the answer sheet or test tape, and for the examiners to run through the test so that it would run smoothly. The qualified physiotherapists were employed at an academic hospital in Cape Town and the physiotherapy students were studying at three universities in the Western Cape. These will be referred to as universities $\mathrm{A}, \mathrm{B}$, and $\mathrm{C}$ in this study. Permission to 
involve the students and clinical staff was granted by the heads of department.

Two test tapes with the sounds in different orders were mixed. They were called test $\mathrm{X}$ and test $\mathrm{Y}$. The tests were randomly allocated to each set of five subjects tested, as five subjects could be tested at once. The subjects listened to the test tape through headphones. Their descriptions of the sounds were marked on the answer sheet as each sound was heard. Each sound was only heard once on the tape. Each sound was heard for at least five breaths. Two test tapes were mixed to prevent subjects tested later having an advantage by conferring with the previously tested subjects. The subjects were informed that there were two tapes being used.

\section{DATA AND DATA ANALYSIS}

Median and mean values for all the samples are presented in the following table:

A non-parametric comparison of two samples (unpaired Mann-Whitney test) was applied to the groups of data to determine if there were any significant differences between the samples. The median was determined to assess the accuracy of the subjects.

When comparing all third year with all fourth year students the $p$ value was 0.0639 (not significant at a $\mathrm{p}$ value > 0.05 ). Comparing fourth year students with qualified physiotherapists proved to be insignificant $(\mathrm{p}=0.3592)$. If each university's students were compared separately, the difference between third and fourth year students proved to be insignificant, with $\mathrm{p}$ values of 0.1521 , 0.1715 and 0.6244 , for universities A, B and $C$ respectively. In assessing the scores of the subjects the authors set the following standards (Figure 3). These standards were set by the authors as there is no research that adequately rates performance in lung sound recognition.

\section{DISCUSSION}

It was shown that the accuracy of physiotherapists does not increase with increasing clinical time. It was also shown that in general physiotherapy students and qualified physiotherapists are minimally accurate in the description of lung sounds. Both the third and fourth year groups attained a median score of 3 out of 6 . The qualified physiotherapists achieved a median of four out of six. However, as it was not statistically sig- nificant, the median score of the entire sample could be seen as representative of the total group. The mean scores for the test increased slightly with increased clinical time. Although this was not significant it is noteworthy and further study in this respect is recommended.

A descriptive analysis of the data was also performed in which specific strengths and weaknesses of the sample groups could be noted and discussed. Each sound will now be presented and specific problems in their identification will be highlighted.

Course crackle (Sound A): All the groups were accurate, except university A, obtaining percentages above 65 . University A only scored $34 \%$. The main problem in all the groups was discerning fine from course crackles.

Pleural Rub (Sound B): 67\% of the qualified group correctly identified this sound and are therefore accurate. The student groups were inaccurate each achieving below $40 \%$. It was clear however that students favoured the term course crackle to describe the sound. In the Wilkins study, only $21.5 \%$ correctly identified a rub, there was also confusion between the terms rub and crackle. ${ }^{13}$

Wheeze (Sound C): The groups scored between 59 and $76 \%$ in describing this sound. This was expected as it is a common sound heard in the clinical setting. In the study by Wilkins, the subjects were $85 \%$ correct in their description of a high pitched wheeze. ${ }^{13}$

Amphoric breathing (Sound D): It was noted that no students at university A correctly described the sound, selecting bronchial breathing in its place. The authors would suggest that the reason for this is that this term was not taught to the students as it is a variant of bronchial breathing. This sound was minimally accurately identified by university $\mathrm{C}$, and inaccurately described by the other universities and qualified group.

Wheeze (Sound E): This sound was correctly identified by most of the subjects. Universities $A$ and $C$ as well as the qualified group scored above $60 \%$. University B scored $43 \%$.

Comparison of left and right lung fields (Sound F): Interestingly students from Universities A B and C performed moderately, minimally and accurately respectively; while qualified staff were inaccurate. Forty percent of the qualified staff incorrectly described the normal lung sound as being harsh or bronchial, and the decreased lung sound as a normal sound. The authors suggest that the inaccuracy displayed by the qualified subjects might be due to the fact that they do not auscultate normal chests as frequently as students do during their training.

The favoured nomenclature is that as described by Forgacs (1978), as opposed to the "American" terminology. In other words crackle and wheeze are more commonly used than rale and rhonchus. Qualified physiotherapists and students at university A used the terms rale and rhonchus more often than the other subjects. A possible explanation is that many of the qualified staff are not recent graduates and could therefore be using the older "American" nomenclature. The possible reason for the use by students is that they have learnt these terms through contact with qualified staff clinically, or that it was simply guessing. It was evident, while marking the tests, that most of the subjects used the Forgacs (1978) nomenclature, only using the term rale and rhonchus once in the test. Thus the "American" nomenclature was used inconsistently and haphazardly, lending weight to our theory that it was guessing.

\section{LIMITATIONS}

A fixed frequency response curve was not performed on the recording and playback apparatus. It is possible that by excluding the stethoscope tubing from the recording apparatus the signal transmission qualities of the stethoscope were changed. This would result in the subjects not hearing the same sound as is heard clinically. The use of headphones could have further influenced the characteristics of the sound.

The volume control on the tape deck during the test was dependent on the subjective recommendations of the respiratory specialists as well as the subjects taking part in the pilot study.

The software package used in the verification of the lung sounds could not perform $\log$ amplitude versus $\log$ frequency plots, thus necessitating the use of other objective criteria.

All the eligible subjects were not tested due to absenteeism on the days when testing was done.

The sample size of the qualified group could have been larger.

All qualified staff were tested irrespective of whether they worked in a car- 
diopulmonary area or not. This may account for the poor results obtained, although on closer inspection of the group of qualified staff tested it was clear that only one of the subjects worked exclusively in a non-respiratory area.

The validity of the study would be increased if all the subjects had received the same tuition in auscultation. In reality all the subjects had received tuition through differing teaching methods as well as having different clinical supervision and time spent on respiratory rotations.

The reliability of the study could have been increased had all the subjects been under more controlled circumstances, for example all the subjects should have been tested in a sound proof environment.

Since the researchers recorded the lung sounds, the study cannot be duplicated without the original tape.

\section{CONCLUSION AND SIGNIFICANCE}

It was found that certain lung sounds are easier to describe compared to others. Subjects fared well when describing crackles and wheezes but fared poorly when describing pleural rubs and amphoric breath sounds. The differentiation of fine and coarse crackles presented problems for some subjects. This is also true in the discrimination between a coarse crackle and a pleural rub. These are areas of concern which may need to be further investigated as the clinical implications are different for each sound. It was noteworthy that students were more accurate in describing a normal breath sound compared to the qualified group.

The results also suggested that there is no significant increase in accuracy with increasing clinical time. A slight trend towards increased accuracy was however noted when comparing the mean scores of the groups.

The authors concluded that the "Forgacs" nomenclature is used by the majority of students and qualified staff tested. ${ }^{14}$

By realising the levels of accuracy existing in lung sound recognition and description, important inroads can be made into teaching methods used. This will have a positive effect on the delivery of health services to the nation as a whole. Excellent auscultation skills supported by a detailed clinical assessment will improve the ability to accurately diagnose chest pathology.

The authors feel that the importance of accuracy in the art of auscultation is of vital importance for the following reasons:

1. As mentioned in the introduction, the swing towards primary health care has the effect of decentralising the health care system. This means that patients will come into contact with health care workers other than specialised respiratory physicians for chest complaints.

2. Access to a stethoscope is comparatively inexpensive and can therefore easily be used in community health centres. The stethoscope is a simple instrument that can be carried easily and has low repair costs.

3. If the health care worker is competent in the art of auscultation there should be no need to refer the patient for expensive diagnostic tests. This will save the health care system money as an early correct diagnosis and treatment will hopefully prevent complications from developing and this will lessen the burden on health care.

4. It is important for the physiotherapist to be competent in auscultation as effctive treatment is partially based on the auscultation findings. An incorrect clinical decision is not only deleterious for the patient but also reduces the efficacy of the therapist as much valuable time is wasted treating these patients inappropriately.

\section{REFERENCES}

1. African National Congress. The Reconstruction and Development Programme: A policy Framework 1994a Umanyano Publications Johannesburg

2. African National Congress. A National Health Plan for South Africa 1994b Bahr Mapping and Printing Lesotho

3. Shannon D. "You See but You do not Observe" Chest 1993, 104: 1320-1321.

4. Baum G and Wolinsky E. (Eds) Textbook of Pulmonary Diseases. Vol. 1. 4th Edition 1989: 221-225 Little, Brown and Company Boston,Toronto.

5. Pasterkamp H, Montgomery $M$ and Wiebicke W. Nomenclature used by Health Care Professionals to Describe Breath Sounds in Asthma. Chest 1987b 92, 2: 346-352.

6. Graviely N, Palti Y and Alroy G. Spectral characteristics of normal breath sounds. Journal of Applied Physiology 1981, 50: 307-313.

7. Murphy R, Holford S and Knowler W. Visual lung sound characterisation by time expanded wave form analysis. The New England Journal of Medicine 1977, 296: 968-971.

8. Munataka M, Ukita H, Doi I, Ohtsuka Y, Masaki Y, Homma Y and Kawakami Y. Spectral and waveform Characteristics of fine and coarse crackles. Thorax 1991, 46: 651-657.

9. Burton G and Hodgkin J. (Eds) Respiratory Care: A Guide to Clinical Practice 2nd Edition 1984: 293-296 JB Lippincott Company, Philidelphia.

10. Mikami R, Murao M, Cugell D, Chrétien J, Cole P, Meier-Sydow J, Murphy R and Loudon R. International Symposium on Lung sounds: Synopsis of Proceedings. Chest 1987, 92: 342-345.

11. Loudon R and Murphy R. State of the art: Lung sounds American Review of Respiratory Diseases 1984, 130: 663-672.

12. American College of Chest Physicians, American Thoracic Society Pulmonary Terms and Symbols: A Report of the ACCP-ATS Joint Committee on Pulmonary Nomenclature. Chest 1975, 67: 583-593.

13. Wilkins R, Dexter J, Murphy R and Delbono E. Lung Sounds Nomenclature Survey. Chest 1990, 98: 886-889.

14. Forgacs P. Lung Sounds 1978 Balliere Tindall, London. 